\section{New strategies for AIDS therapy and prophylaxis}

SIR-In search for a reactive agent against HTLV-III/LAV, the virus that causes AIDS (acquired immune deficiency syndrome), the attention is focused on neutralizing antibodies. Such antibodies have been detected in the blood of AIDS patients ${ }^{1,2}$ and form the starting point for the production of vaccines ${ }^{3}$. However, there are as yet no indications that these antibodies influence the course or the outcome of the disease. Therefore, alternative strategies should be pursued. A recently developed technique for specific inhibition of gene expression provides one possible new way to tackle the AIDS virus. It is based on the inhibition of the messenger RNA translation through the interaction of anti-sense RNA with a specific messenger and in several systems has been shown to be very successful ${ }^{4-6}$. Accordingly, the introduction of an actively transcribed template for antisense RNA of one of the AIDS virus genes into infected cells may induce inhibition of virus replication and increase survival chances of the patient.

The construction of such a template will not be difficult, but how is it to be delivered to the correct target cells? The answer is, in principle, simple. Make a recombined AIDS virus in which one of the genes is in the reversed orientation. Infection of a patient with this virus will ensure that the recombined genome is delivered precisely to those cells that are sensitive to, or harbour, the AIDS virus. Cells not yet invaded by the AIDS virus may be protected by the recombinant antisense RNA against integration of the AIDS genome. In this way spreading of the virus within the body could be prevented. Which segment of the genome of the virus should be reversed? The gag or pol regions are unattractive, because the corresponding genes of any helper virus involved in the production of the recombinant might be blocked as well. With the reversion of the env gene there is the additional problem that the recombinant virus might acquire the envelope of the helper, resulting in the infection of the wrong target cells. A fourth gene, tat, which has recently been identified on the genome of the AIDS virus ${ }^{78}$, is more promising. The gene product is a trans-activator of viral transcription. Preventing the synthesis of the tat protein results in a significant reduction of virus replication, which is exactly what we are looking for. In fact, reversion of the second exon of the tat gene, which is located between pol and env, would alone be sufficient.

In theory, the only problem that we are left with is the efficient production of the recombinant virus, because this may require the action of the trans-activator protein. A cell line producing and accumulating the tat protein in sufficient quantity. prior to infection with the recombinant virus, might be effective. Now that the sequence of the gene is known such a cell line is within reach. Alternative methods can be thought of, one of which is to load cells before infection with the tat-protein, obtained from any source, by the method of scrape loading ${ }^{9}$, which works well especially with proteins of small size.

Although problems would surely show up during the development of the strategy outlined above, AIDS is too serious a threat to human life to let any possible means for therapy and prophylaxis go untested. The method might also be tried for other diseases, including the T-cell leukaemias caused by HTLV-I and -II.

E.C.M. MARIMAN

Department of Human Genetics,

University of Nijmegen,

6525 GA Nijmegen,

The Netherlands

1. Weiss, R A. et al Nature 316, 69 (1985)

2. Robert-Guroff, M Nature 316, 72 (1985).

. Crowl. R. et al. Cell 41, 979 (1985).

Izant, J. G \& Weintraub, H. Science 229, 345 (1985)

Melton, D. A. Proc natn. Acad. Sct. U.S.A. 82, 144 (1985)

Rosenberg, U. B. et al. Nature 313, 7(1)3 (1985)

. Arya, S. K. et al. Science 229, 69 (1985)

. Sodroskı, J. et al. Sctence 229, 74 (1985)

9. McNeil, P. L. \& Taylor, D. L. Cell Calcium 6, 83 (1985)

SIR-We wish to suggest a gene therapy scheme for the control of acquired immune deficiency syndrome (AIDS). The current view on AIDS is that infection with HTLV-III/LAV virus, trophic for OKT4 $4^{+}$lymphocytes, results in breaking the backbone of the immune system. The diversity of viral strains and the nature of the infection renders problematic the production and use of vaccines'. A strategy has been proposed consisting of "protecting" T-cell clones against HTLV-III by using viral interference through infection with attenuated virus ${ }^{2}$.

In our view a better way to achieve this protection would be through the strategy of antisense RNA. Inhibition of gene expression has been achieved in a variety of systems with as little as 52-base-pair, homology to the $5^{\prime}$ untranslated segment of the sense RNA'. Helper-free, defective HTLV-III, containing the appropriate group-specific inverted viral gene segments could be produced in a packaging cell line modelled on the Zipneo- $\psi 2$ system ${ }^{+}$. To ensure out-titration of senseRNA, a gene amplification system, such as the DHFR gene responsive to the anticancer drug methotrexate, could be built into this antisense virus'.

AIDS therapy would consist in removal of bone marrow stem cells from patients. followed by their infection with the antivirus and reinsertion, resulting in the production of "protected" lymphocytes. Alternatively, peripheral blood lymphocytes obtained by pheresis would be infected and reinserted. Methotrexate induced amplification of the antisense provirus may be carried out in vitro, as needed". By these means a critical and sufficient population of $T$ cells might be protected so that an effective immune response against all strains of HTLV-III and against opportunistic infections could be achieved.

RAYMOND TELLIER JOSEPH M. WEBER

Department of Microbiology, Centre Hospitalier Universitaire de Sherbrooke,

Québec, Canada J1H 5 N4

1. Wong-Staal et al. Sctence 229, 759 (1985).

2. Bolognesı, D. P. \& Fischinger P. J. Cancer Res. 45, 4700s (1985).

Izant, J. G \& Weintraub, H. Science 229, 345 (1985).

4. Cepko, C. L. et al. Cell. 37, 1053 (1984)

Miller, A. D. et al. Mol Cell. Btol. 5, 431 (1985)

6. Schornaget, J H. \& McVıe. J. G. Cancer Treat Rev 10, 53 (1983)

\section{Cerebral supermanifolds and little brains}

SiR-John Marshall's analysis of current neuropsychological efforts to correlate specific lexical dysfunctions with "focal" brain damage raises, expectedly, some presently unfocused issues: (1) The "focal" brain damage reported by Hart et al. was a "left-hemisphere cerebrovascular accident" diagnosed by computerized tomography as "an infarction involving the left frontal-lobe and basal ganglia." This is, by cerebral neuroanatomical criteria, a truly enormous, almost global region of damage.

(2) Marshall's interesting quotation from Johann Gesner ${ }^{2}$ suggests that even the classical eighteenth century allowed speculation about the validity of linear transformations from a 'psycho'-logical to a cartesian frame of reference in cerebral cortex.

(3) Despite some rough approximations to a two-dimensional somatotopy found in mammalian and primate somatosensoryspecific cortex (for example, the primate digit and mouse whisker-barrel "representations"), we have no reason to suppose a linear two- or three-dimensional mapping of inner and outer spacetime to exist inside our heads. Since the days of Maxwell to Einstein would it not be more reasonable to use differential geometry in our approach to understanding how brains might work? A start has already been made for our "little brains""

Hilton STOWELL

ERBP Laboratory,

120 Nature Creek SW,

Milledgeville Georgia 31061, USA

1. Hart. J.. Berndt. R S. \& Caramazza, A Nature 316, 439 (1985).

(.C Nature 316, 388 (1985)

3. Pelhonusz. A. \& Llinás, R Neuroscience (1977-85).

\section{Scientific Correspondence}

Scientific Correspondence is intended to provide a forum in which readers may raise points of a rather technical character which are not provoked by articles or letters previously published (where the Matters Arising section remains appropriate) 